\title{
Motion Opponency in Visual Cortex
}

\author{
David J. Heeger, ${ }^{1}$ Geoffrey M. Boynton, ${ }^{1}$ Jonathan B. Demb, ${ }^{1}$ Eyal Seidemann, ${ }^{2}$ and William T. Newsome ${ }^{2}$ \\ ${ }^{1}$ Department of Psychology, and ${ }^{2}$ Howard Hughes Medical Institute and Department of Neurobiology, Stanford University, \\ Stanford, California 94305-2130
}

Perceptual studies suggest that visual motion perception is mediated by opponent mechanisms that correspond to mutually suppressive populations of neurons sensitive to motions in opposite directions. We tested for a neuronal correlate of motion opponency using functional magnetic resonance imaging (fMRI) to measure brain activity in human visual cortex. There was strong motion opponency in a secondary visual cortical area known as the human MT complex $(\mathrm{MT}+)$, but there was little evidence of motion opponency in primary visual cortex. To determine whether the level of opponency in human and monkey are comparable, a variant of these experiments was performed using multiunit electrophysiological recording in areas
MT and MST of the macaque monkey brain. Although there was substantial variability in the degree of opponency between recording sites, the monkey and human data were qualitatively similar on average. These results provide further evidence that: (1) direction-selective signals underly human MT+ responses, (2) neuronal signals in human MT+ support visual motion perception, (3) human MT+ is homologous to macaque monkey MT and adjacent motion sensitive brain areas, and (4) that fMRI measurements are correlated with average spiking activity.

Key words: MT; V1; neuroimaging; fMRI; motion; visual motion perception; motion opponency; vision; visual cortex
Computational theories of visual motion perception typically include a motion opponent stage in which, for example, the response of a hypothetical leftward-selective neuron is subtracted from that of a rightward-selective neuron to yield a neuronal signal for net rightward motion (van Santen and Sperling, 1984, 1985; Qian et al., 1994b; Adelson and Bergen, 1985; Simoncelli and Heeger, 1998). The importance of opponent mechanisms for motion perception is supported by a number of perceptual studies (Levinson and Sekuler, 1975a; Mather and Moulden, 1983; van Santen and Sperling, 1984; Stromeyer et al., 1984; Lubin, 1992; Qian et al., 1994a; Zemany et al., 1998). For example, superimposing two identical sinusoidal grating patterns moving in opposite directions produces what is called a counterphase grating. The counterphase grating appears to flicker in place with no net motion, as if the motions of the two component gratings canceled one another. Likewise, when a pair of superimposed random dot fields are paired so that each dot moving in one direction is always located near a dot moving in the opposite direction, they appear to flicker (Qian et al., 1994a). However, the results of some other perceptual studies suggest that opposite directions of motion are processed independently, inconsistent with oppo-

Received Dec. 22, 1998; revised June 1, 1999; accepted June 3, 1999.

G.M.B. supported by a National Institute of Mental Health (NIMH) postdoctoral research fellowship. D.J.H. supported by an NEI grant (R01-EY11794) and an NIMH grant (R29-MH50228). W.T.N. is an Investigator of the Howard Hughes Medical Institute.

Special thanks to G. H. Glover (and the Richard M. Lucas Center for Magnetic Resonance Spectroscopy and Imaging, supported by an National Institutes of Health National Center for Research Resources grant) for technical support.

Correspondence should be addressed to David Heeger, Department of Psychology, Stanford University, Stanford, CA 94305-2130.

Dr. Boynton's present address: The Salk Institute for Biological Studies, 10010 North Torrey Pines Road, La Jolla, CA 92037-1099.

Dr. Demb's present address: Department of Neuroscience, University of Pennsylvania Medical School, Philadelphia, PA 19104-6058.

Dr. Seidemann's present address: Department of Neurobiology, Weizmann Institute of Science, Rehovot 76100, Israel.

Copyright (C) 1999 Society for Neuroscience 0270-6474/99/197162-13\$05.00/0 nency (Levinson and Sekuler, 1975b; Watson et al., 1980; Raymond and Braddick, 1996).

Motion opponency is believed to play an important role in the response properties of neurons in the middle temporal area (MT or V5) of the monkey brain, a region of visual cortex that is widely viewed as a cornerstone of the neuronal pathways subserving visual motion perception (Dubner and Zeki, 1971; Zeki, 1974; Albright, 1993). Neuronal signals that carry motion information pass from the primary visual cortex (V1) to MT, and then to adjacent motion-sensitive visual areas, including areas MST and FST (Maunsell and Van Essen, 1983; Ungerleider and Desimone, 1986; Krubitzer and Kaas, 1990; Boussaoud et al., 1990; Movshon and Newsome, 1996). MT neurons respond vigorously to a visual pattern moving in a preferred direction, but the responses can be suppressed substantially by superimposing a second pattern moving in a nonpreferred direction (Snowden et al., 1991; Bradley et al., 1995). This suppression is particularly strong for paired dot patterns (Qian and Andersen, 1994).

Human visual cortex contains an area in the lateral portion of the occipital lobe $(\mathrm{MT}+$, or V5) that may be homologous to monkey MT and adjacent motion-sensitive areas such as MST and FST (Zeki et al., 1991; Tootell and Taylor, 1995). The case for homology between monkey MT and human MT + rests on its general location with respect to other identified visual areas in both species, its cytoarchitecture, and its heightened sensitivity to low-contrast, moving stimuli in comparison to other visual areas (see Discussion for additional references). In the monkey, however, the primary physiological signature of MT is direction selectivity measured at the single neuron and columnar levels (Zeki, 1974; Maunsell and Van Essen, 1983). No single neuron or columnar level observations have yet been made for human $\mathrm{MT}+$. One neuroimaging study has provided indirect evidence for directional interactions in human $\mathrm{MT}+$, based on functional magnetic resonance imaging (fMRI) measurements of the motion aftereffect (Tootell et al., 1995b). Thus, the proposed homology 
with monkey MT must be regarded as somewhat tentative until more detailed physiological and anatomical data are acquired.

In the current study, we tested for a neuronal correlate of motion opponency in the human visual cortex using fMRI (Ogawa et al., 1990, 1992; Belliveau et al., 1991; Kwong et al., 1992). The goals of the study were twofold: (1) to determine whether functional activity in human MT + reflects motionopponent mechanisms (and by inference, directionally selective mechanisms), and (2) to compare motion-opponent responses in human MT+ with motion-opponent responses in monkey MT to gain further evidence concerning potential homologies between the two species.

\section{MATERIALS AND METHODS \\ fMRI}

Each subject participated in several MR scanning sessions: one to obtain a standard, high-resolution, anatomical scan, one to identify visual areas $\mathrm{V} 1$ and $\mathrm{MT}+$, and several sessions (10 for subject gmb and 11 for subject djh) to measure fMRI responses in the various experimental conditions. Each subject repeated each experiment between four and eight times in separate fMRI scans. The figures below plot the mean and SE of these repeated measurements.

Moving gratings versus counterphase gratings. Brain activity was measured while subjects viewed moving sinusoidal gratings and counterphase (or contrast-reversing) gratings. Counterphase gratings were presented for $18 \mathrm{sec}$ followed by $18 \mathrm{sec}$ of moving gratings (each $18 \mathrm{sec}$ half-cycle was divided into nine $2 \mathrm{sec}$ "trials," as described below). During each 252 sec fMRI scan, this counterphase/moving cycle was repeated seven times. The contrast of counterphase gratings was twice that of the moving gratings (where contrast was defined in the usual way as the peak luminance of the stimulus minus the minimum luminance, divided by twice the mean), i.e., the counterphase gratings were constructed by superimposing a pair of moving gratings that moved in opposite directions.

We used several different combinations of spatial frequencies $(0.4$ cycle $/^{\circ}$ and 0.8 cycle $^{\circ}$ ), temporal frequencies ( 4 and $8 \mathrm{~Hz}$ ), and mean luminances $\left(3\right.$ and $\left.36 \mathrm{~cd} / \mathrm{m}^{2}\right)$. Stimulus contrasts varied slightly within each trial, as described below, so that subjects could perform a contrast discrimination task. The chosen contrasts varied slightly above and below either 6 or $45 \%$ for the moving gratings, and 12 or $90 \%$ for the counterphase gratings (Table 1).

MT+ activity can be modulated by attention (Corbetta et al., 1990; O'Craven et al., 1997; Beauchamp et al., 1997; Gandhi et al., 1999). To control for attentional state, subjects performed a contrast discrimination task throughout each fMRI scan. Each 2 sec trial consisted of a pair of $500 \mathrm{msec}$ stimulus presentations, one with slightly higher contrast than the other, separated in time by a $200 \mathrm{msec}$ blank interval, followed by an $800 \mathrm{msec}$ response interval. A uniform gray field (equal to the mean luminance of the grating stimuli) was presented during the blank and response intervals. During the response interval, subjects pressed one of two buttons to indicate which of the two preceding stimuli appeared to have the higher contrast. Feedback was provided after each trial. Subjects practiced the task extensively until their performance reached asymptotic levels. The contrast increments that were used during the fMRI scans were chosen based on these asymptotic performance levels so that subjects would perform with an accuracy of $\sim 80-90 \%$ correct (Table 1 ).

Because MT+ activity can exhibit a motion aftereffect (Tootell et al., 1995b), the stimuli were designed to minimize visual adaptation. Specifically, alternating brief stimulus presentations with blank intervals minimized any effects of contrast-dependent adaptation by visual neurons. In addition, the gratings alternated orientation on every trial (rightward or leftward diagonal), to minimize orientation- and direction-specific adaptation.

Human MT+ is believed to be homologous to a collection of macaque monkey brain areas, including some (e.g., MST) in which neuronal activity can be modulated by eye movements (Newsome et al., 1988). This would present a possible alternative explanation of our results if subjects had moved their eyes when viewing the moving gratings. To minimize eye movements, subjects fixated a small, high-contrast fixation mark that was displayed continuously throughout each scan. In addition, for the moving gratings, the grating stimulus was bisected along a diagonal line parallel to the orientation of the grating bars, and each half
Table 1. Contrast discrimination performance for grating experiments

\begin{tabular}{|c|c|c|c|}
\hline \multirow[b]{2}{*}{ Condition } & \multirow[b]{2}{*}{ Contrasts } & \multicolumn{2}{|c|}{$\%$ correct } \\
\hline & & djh & $\mathrm{gmb}$ \\
\hline \multicolumn{4}{|l|}{ Figure 2 (circles) } \\
\hline Moving & $41.5-50 \%$ & 78 & 90 \\
\hline Counterphase & $83-100 \%$ & 82 & 89 \\
\hline \multicolumn{4}{|l|}{ Figure 2 (squares) } \\
\hline Moving & $38.5-50 \%$ & 87 & 93 \\
\hline Counterphase & $77-100 \%$ & 83 & 85 \\
\hline \multicolumn{4}{|c|}{ Figure 2 (triangles) } \\
\hline Moving & $5.96-6.56 \%$ & 97 & 81 \\
\hline Counterphase & $11.9-13.1 \%$ & 77 & 64 \\
\hline \multicolumn{4}{|l|}{ Figure 3} \\
\hline Moving & $38.5-50 \%$ & 94 & 93 \\
\hline Counterphase & $77-100 \%$ & 93 & 93 \\
\hline \multicolumn{4}{|l|}{ Figure 4} \\
\hline Med contrast & $45.5-50 \%$ & 74 & 63 \\
\hline High contrast & $90.1-100 \%$ & 87 & 86 \\
\hline
\end{tabular}

of the grating moved toward the fixation mark. Thus, the overall motion of the grating served to "draw" the eyes toward the fixation point, avoiding a single, powerful optokinetic stimulus.

The stimuli were displayed on a screen made of rear-projection material, positioned at the opening of the bore of the magnet near the subjects' knees. The subjects, lying on their backs, looked directly up into an angled mirror to see the rear-projection screen. The display subtended $14 \times 14^{\circ}$ of visual angle.

Moving/counterphase gratings versus blank. In one series of scans, we measured brain activity while the stimuli alternated between $18 \mathrm{sec}$, during which moving gratings $\left(0.4\right.$ cycle $/{ }^{\circ}, 8 \mathrm{~Hz}, 3 \mathrm{~cd} / \mathrm{m}^{2}$, contrast varied slightly around $44.25 \%$ ) were presented followed by $18 \mathrm{sec}$ during which the screen was a uniform gray field (equal to the mean luminance of the gratings). In a second series of scans, the stimuli alternated between counterphase gratings (same spatial and temporal frequency, contrast varied slightly around $88.5 \%$ ) and a uniform gray field. The gratings were again presented in a series of $500 \mathrm{msec}$ intervals while subjects performed a contrast discrimination task. The orientations of the gratings were again alternated on every trial (rightward/leftward diagonal). For the moving gratings, the two halves of the screen again moved toward the fixation mark. Subjects did not perform a task during the half-cycles when no stimuli (gray fields) were presented. Hence, we did not completely control subjects' attention during these scans.

Supersaturation control. Brain activity was measured while moving grating stimuli $\left(0.8 \mathrm{cycle} /{ }^{\circ}, 4 \mathrm{~Hz}, 36 \mathrm{~cd} / \mathrm{m}^{2}\right)$ were presented. The gratings were again presented in a series of $500 \mathrm{msec}$ intervals while subjects performed a contrast discrimination task, $18 \mathrm{sec}$ at high contrasts (varied slightly around $95 \%$ ) followed by $18 \mathrm{sec}$ at medium contrasts (varied slightly around $47.75 \%$ ). The gratings' orientations were again alternated on every trial (rightward/leftward diagonal), and the two halves of the screen again moved toward the fixation mark.

Paired dots versus unpaired dots. Brain activity was measured while subjects viewed paired and unpaired dot patterns. Dots were square in shape, $0.1^{\circ}$ across, and white $\left(120 \mathrm{~cd} / \mathrm{m}^{2}\right)$ on a black background (4 $\left.\mathrm{cd} / \mathrm{m}^{2}\right)$. The dot density was varied to be relatively sparse $(\sim 200$ dots uniformly distributed across the $20 \times 20^{\circ}$ field) or relatively dense ( $\sim 2400$ dots). Half the dots moved to the right, and the other half moved to the left with an average speed of $2.5^{\circ} / \mathrm{sec}$. Following Qian and Andersen (1994), each pair of dots in the paired dot patterns started at a separation of $0.19^{\circ}$ on average, moved toward and across each other, and was replotted at a new randomized location when the separation was again $0.19^{\circ}$ on average. The dots, therefore, had an average lifetime of $150 \mathrm{msec}$ before being replotted. The start/end positions and lifetime were randomized by $\pm 5 \%$ so that subjects could not use the initial/final dot separation as a cue to the dot speed. The replotting of the dot pairs was asynchronous with respect to each other. There was a blank disc with no dots in the central region $\left(2^{\circ}\right.$ radius) around the fixation mark. The unpaired dot patterns were identical to the paired ones, except that the 
two dots in each pair were positioned independently and randomly. The unpaired dots thus appeared to move past one another like two semitransparent surfaces, whereas the paired dots appeared to flicker.

Subjects performed a speed discrimination task. Each $2 \mathrm{sec}$ trial consisted of a pair of $500 \mathrm{msec}$ stimulus presentation intervals. The dots moved with a base speed in one interval and a slightly faster speed in the other interval. The screen was black between the stimulus presentations. Subjects pressed a button to indicate which of the two preceding stimuli appeared to move faster. Feedback was provided after each trial. Subjects practiced the task extensively until their performance reached asymptotic levels. The speed increments that were used during the fMRI scans were chosen based on these asymptotic performance levels so that subjects would perform with an accuracy of $\sim 75-80 \%$ correct (Table 2 ).

The stimulus alternated between 18 sec during which paired dots were presented followed by $18 \mathrm{sec}$ during which unpaired dots were presented. During each $252 \mathrm{sec}$ fMRI scan, this paired/unpaired cycle was repeated seven times.

The stimuli were displayed on a flat-panel display (NEC, multisynch LCD 2000) positioned just beyond the end of the patient bed. The display was viewed through binoculars (Optolyth-Optik Alpin $8 \times 30$ ) specially modified with all the steel parts removed and replaced with berylliumcopper or brass. A pair of mirrors, angled at $\sim 45^{\circ}$, were attached to the binoculars just beyond the two objective lenses, to enable the subjects to see the LCD display.

Data acquisition. MR imaging was performed on a standard clinical GE 1.5 T Signa scanner with either a GE head coil (grating experiments on subject djh) or a custom designed dual surface coil (grating experiments on subject gmb, dots experiments on both subjects). The experiments were undertaken with the written consent of each subject and in compliance with the safety guidelines for MR research.

Each fMRI scanning session began by acquiring a set of low-resolution, sagittal, anatomical images used for slice selection. Eight adjacent planes were selected with the most ventral slice positioned along the boundary between the occipital lobe and the cerebellum. Approximately the same slices were chosen in each scanning session. A set of structural images were then acquired using a T1-weighted spin echo pulse sequence (500 msec repetition time, minimum echo time, $90^{\circ}$ flip angle) in the same slices and at the same resolution as the functional images. These inplane anatomical images were registered to the high-resolution anatomical scan of each subject's brain so that all MR images (across multiple scanning sessions) from a given subject were aligned to a common threedimensional coordinate grid. Then a series of fMRI scans were performed using a spiral $\mathrm{T} 2 *$-sensitive gradient-recalled echo pulse sequence (1500 msec repetition time, $40 \mathrm{msec}$ echo time, $90^{\circ}$ flip angle, two interleaves, inplane resolution $=1.02 \times 1.02 \mathrm{~mm}$, slice thickness $=4$ $\mathrm{mm}$ ) (Noll et al., 1995; Glover and Lai, 1998). Spiral fMRI pulse sequences compare favorably with echo-planar imaging in terms of spatial resolution and sensitivity (Sawyer-Glover and Glover, 1998).

A bite bar stabilized the subjects' heads. The time-series of fMRIs from each scan were visually inspected for head movements. No post hoc motion correction was applied because there was no indication of head movements during any of the scans.

Data analysis. Each fMRI scan lasted $252 \mathrm{sec}$. Data from the first $36 \mathrm{sec}$ cycle were discarded: (1) to minimize effects of magnetic saturation, (2) to minimize effects of visual adaptation, and (3) to allow time for subjects to practice the task. During the remaining six cycles of each scan, 72 functional images (one every $3 \mathrm{sec}$ ) were recorded for each slice. For a given fMRI voxel (corresponding to a $1 \times 1 \times 4 \mathrm{~mm}$ brain volume), the image intensity changed over time and comprised a time series of data.

The data were analyzed separately in each of two identifiable visual areas, $\mathrm{V} 1$ and $\mathrm{MT}+$. We computed the fMRI response amplitudes and phases by: (1) removing the linear trend in the time series, (2) dividing the time series of each voxel by its mean intensity, (3) averaging the resulting time series over the set of voxels corresponding to the stimulus representation within a visual area (V1 or MT+), and then (4) calculating the amplitude and phase of the best fitting $36 \mathrm{sec}$ period sinusoid. The first step (removing the linear trend) compensates for the fact that the fMRI signal tends to drift, for unknown reasons, very slowly over time. The second step converts the data from arbitrary image intensity units to units of percent signal modulation; this is especially important because the mean image intensity varies substantially with distance from the surface coil. Finally, we computed the vector average and SD of the responses (amplitudes and phases) across measurements that were repeated in separate scans.

Localizing V1. Following well-established methods (Schneider et al.,
Table 2. Speed discrimination performance for paired and unpaired dots

\begin{tabular}{lllll} 
Subject & $\begin{array}{l}\text { Dot } \\
\text { density }\end{array}$ & Stimulus & $\begin{array}{l}\text { \% speed } \\
\text { increment }\end{array}$ & $\%$ correct \\
\hline djh & Low & Paired & 18 & 78 \\
& & Unpaired & 12 & 83 \\
& High & Paired & 18 & 78 \\
& & Unpaired & 12 & 81 \\
gmb & Low & Paired & 18 & 66 \\
& \multirow{3}{*}{ High } & Unpaired & 11 & 75 \\
& & Paired & 27 & 72 \\
& & Unpaired & 18 & 76 \\
\hline
\end{tabular}

$\overline{\text { Speed increments were larger for paired than for unpaired dots, but percent correct }}$ performance was about the same.

1993; Engel et al., 1994; Sereno et al., 1995; DeYoe et al., 1996; Engel et al., 1997), the polar angle component of the retinotopic map was measured by recording fMRI responses as a stimulus rotated slowly (like the second hand of a clock) in the visual field. To visualize these retinotopy measurements, a high-resolution MRI of each subject's brain was computationally flattened (Engel et al., 1997). Area V1 within each hemisphere was identified as a large region of cortex in/near the calcarine sulcus with a retinotopic map spanning half the visual field.

Localizing MT+. Following previous studies (Zeki et al., 1991; Watson et al., 1993; Tootell et al., 1995a), area MT+ was identified based on fMRI responses to stimuli that alternated in time between moving and stationary dot patterns. The dots (small white dots on a black background) moved $\left(10^{\circ} / \mathrm{sec}\right)$ radially inward and outward for $18 \mathrm{sec}$, alternating direction once every second. Then the dot pattern was stationary for the next $18 \mathrm{sec}$. This moving/stationary cycle was repeated seven times. We computed the cross-correlation between the time series of each fMRI voxel and a sinusoid with the same $(36 \mathrm{sec})$ temporal period. We drew MT + regions by hand around contiguous areas of strong activation, beyond the retinotopically organized visual areas. The MT+ regions were confined almost entirely within a single sulcus in each hemisphere. Brain atlases typically do not provide a name for this sulcus but it is easily identified in coronal slices as the sulcus between the inferior occipital sulcus and the lateral occipital sulcus.

Reference scans. The procedures to define V1 and MT+ were performed only once per subject. Because the fMRI data recorded during successive scanning sessions in a given subject were all aligned to a common three-dimensional coordinate grid (see above), we could localize both areas across scanning sessions.

The V1 and MT+ regions were further restricted based on responses to a reference stimulus. The reference scan responses were used to exclude unresponsive voxels, e.g., brain regions that would have responded to visual field locations outside the $14 \times 14^{\circ}$ stimulus aperture, and voxels that had too little overlap with gray matter. The reference stimulus was the same moving versus stationary dot pattern used to localize area $\mathrm{MT}+$. A reference scan was run during each scanning session, usually as the first fMRI scan of the session. Voxels that were unresponsive in the reference scans were discarded in the analysis of all subsequent scans in that scanning session. Responsive voxels were defined as those that were strongly correlated ( $r>0.4$ and $1-5 \mathrm{sec}$ time lag) with a $36 \mathrm{sec}$ period sinusoid. Excluding voxels based on the reference scan decreased the variability in the data, but it was not critical for any of our conclusions; when this step in the analysis was skipped, all but one of the statistically significant results reported below retained statistical significance, and many of the results attained the same significance level. Even the one result that did fall below significance threshold (see Fig. 2, subject gmb, filled circle) remained close $(p=0.059)$.

Statistics. One-tailed $t$ tests were used to determine the statistical significance of the measured modulations in brain activity. First, we computed the fMRI response amplitude and phase for each repeat of each experiment (see above). Second, for each subject and for each visual area, the responses to the aforementioned reference scans were averaged across scanning sessions. Third, we computed the component of the fMRI responses with zero phase lag relative to the responses from the reference scans. Fourth, we computed the mean and SE of the resulting response amplitude components. Finally, we tested the null hypothesis 

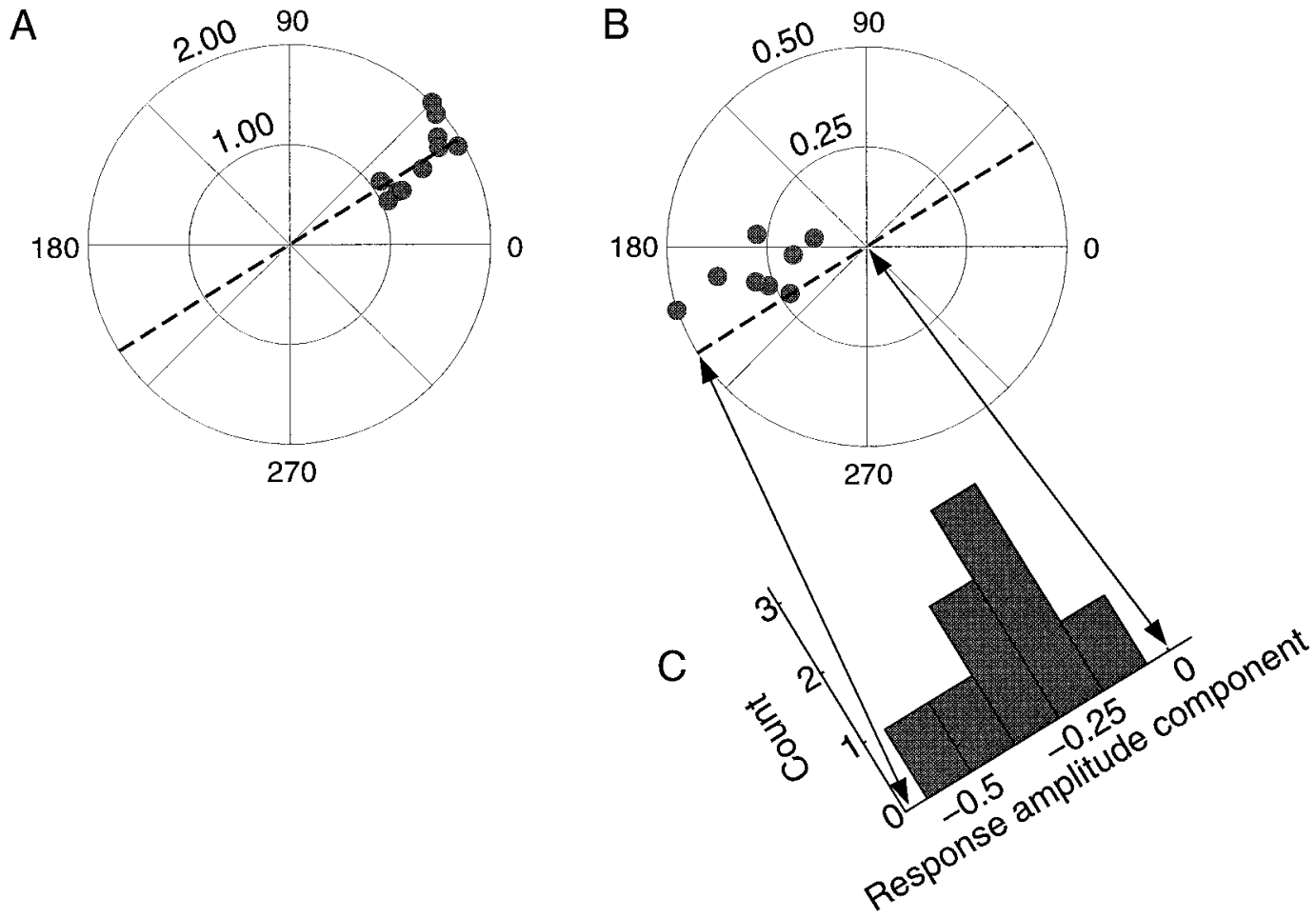

Figure 1. Statistical analysis of fMRI measurements. A, fMRI measurements of MT + activity from 10 repeats (1 per scanning session) of the reference scan for subject djh. Response amplitude (percent MR signal modulation) indicated by radial distance from the origin and response temporal phase indicated by the angle from the horizontal axis. Circles, Responses from the 10 individual scans. Dashed line indicating the reference phase, passes through the vector mean of the 10 data points. $B, \mathrm{MT}+$ responses in one of the experimental test conditions (high-density paired versus unpaired dots) for the same subject. Circles, Responses from the eight individual scans. Dashed line (copied from $A$ ) indicates the reference phase. $C$, Histogram of fMRI response amplitude components, produced from $B$ by computing the orthogonal projection of each data point onto the dashed line.

that the mean response amplitude component was zero, i.e., that there was no modulation of brain activity.

This procedure is illustrated in Figure 1. Figure $1 A$ plots fMRI measurements of MT + brain activity from 10 repeats of the reference scan, one per scanning session. In the polar plot, fMRI response amplitudes are represented by the radial distances from the origin, and fMRI response phases are represented by the angles counterclockwise from the horizontal axis. The dashed line passes through the vector mean of the data points. We refer to the angle of the dashed line as the reference phase.

The reference phases in V1 were 29 and $31^{\circ}$ for subjects djh and gmb, respectively. In MT + the reference phases were 29 and $33^{\circ}$ for subjects djh and gmb, respectively. Given that a $10^{\circ}$ phase lag in our $36 \mathrm{sec}$ period paradigm corresponds to a $1 \mathrm{sec}$ delay, these reference phases are consistent with the 2-4 sec temporal lag that is characteristic of the hemodynamic delay (Boynton et al., 1996; Malonek and Grinvald, 1996, 1997).

Figure $1 B$ is a polar plot of MT+ responses in one of the experimental test conditions (high-density paired versus unpaired dots). The dashed line in Figure $1 B$ is copied from Figure $1 A$, i.e., it again indicates the $\mathrm{MT}+$ reference phase for that subject.

Figure $1 C$ plots a histogram of response amplitude components. The histogram was produced from Figure $1 B$ by computing the orthogonal projection of each data point onto the dashed line that indicates the reference phase. Assuming that the reference phase is an accurate estimate of the hemodynamic delay, the mean of the resulting histogram is an unbiased estimate of the true (noise-free) response amplitude. Statistical tests (one-tailed $t$ tests) were computed based on the mean and $\mathrm{SE}$ of the response amplitude components, after projecting onto the reference phase line in this way.

This procedure takes full advantage of the a priori knowledge of the periodic design of the experiments. For example, if MT + had responded more strongly to the paired dots in the first half of each temporal cycle, then the responses would have been in the top right quadrant of the polar plot. Instead, MT+ responded more strongly to the unpaired dots in the second half of each temporal cycle, so the responses are in the bottom left quadrant of the polar plot. Any data points that do not lie near the reference phase line must be dominated by measurement noise.

Converting the bivariate (amplitude and phase) responses into univariate response amplitude components enabled us to perform a standard statistical test, but it was not critical for any of our conclusions. Our conclusions are supported equally well using statistics on the bivariate distributions of response amplitudes and phases, like the example shown in Figure $1 B$. The circles in Figures 2, 4, and 5 represent $95 \%$ confidence intervals on the bivariate response distributions.

For the bar graph in Figure 3, each bar indicates the mean and SE of the response amplitude components, after projecting (as shown in Figure 1) the data onto the reference phase line. The response phases for the data plotted in Figure 3 were all within $20^{\circ}$ of one another. Hence, although it was convenient to plot the response amplitude components, our conclusions are equally well supported by the bivariate response amplitudes and phases.

\section{Electrophysiology}

Electrophysiological experiments were conducted in order to obtain data concerning motion opponency in monkey MT that could be reasonably compared to the fMRI data obtained in humans. Our goal was to estimate the overall level of activity in MT (which is what the fMRI signal is thought to reflect), by pooling activity from numerous recording sites in MT measured one at a time. Our electrophysiological methods therefore departed from standard recording techniques in three important ways. First, instead of measuring the activity of single MT units, we focused primarily on multiunit recordings that reflect summed activity of a population of neurons near the recording electrode. Second, we attempted to obtain an unbiased sample of recording sites in MT. Thus, we made long penetrations through MT, recording neuronal activity at fixed intervals along each penetration (every 150-200 $\mu \mathrm{m}$ ) irrespective of the exact physiological properties of each site. In one animal, we recorded single units in addition to multiunit activity whenever possible, but the recording sites were not selected specifically to isolate single units. 
Finally, instead of tailoring the visual stimulus to the response properties of the recorded neurons (optimized for receptive field location, preferred spatial and temporal frequency, etc.), we tested each recording site with a fixed battery of visual stimuli similar to those used in the fMRI study.

Behavioral task and visual stimulus. Two rhesus monkeys (one male and one female) were trained to fixate a central spot on a computer monitor while viewing either a moving sinusoidal grating or counterphase grating. The monkey's eye position was measured using a scleral search coil system (CNC Engineering).

Visual stimuli were moving gratings and counterphase gratings with the same spatial frequency $\left(0.8 \mathrm{cycle}^{\circ}\right)$ and temporal frequency $(4 \mathrm{~Hz})$. The contrast of the moving gratings was either 3 or $50 \%$, and the contrast of the counterphase gratings was 6 or $100 \%$. The gratings subtended $14 \times$ $14^{\circ}$ of visual angle and were centered on the fixation point as in the fMRI experiments. Grating stimuli were presented on a uniform gray background of the same mean luminance $\left(50 \mathrm{~cd} / \mathrm{m}^{2}\right)$. Receptive fields at all our MT recording sites lay completely or partially inside the stimulus aperture.

Each trial began with the appearance of a small central fixation point. Throughout each trial, which lasted $6.3 \mathrm{sec}$, the monkey was required to maintain fixation within a small window $\left(3 \times 3^{\circ}\right.$ or smaller $)$ around the fixation point. Trials in which the monkey broke fixation prematurely were aborted without reward and were excluded from our analysis.

Each trial lasted $6.3 \mathrm{sec}$, beginning with a $0.5 \mathrm{sec}$ fixation interval. After the initial fixation interval, a series of six $0.5 \mathrm{sec}$ stimulus intervals alternated with $0.5 \mathrm{sec}$ blank intervals. The final blank interval was shortened to $0.3 \mathrm{sec}$. We alternated brief stimulus presentations with blank intervals in this way to minimize any effects of contrast-dependent adaptation, and so that the stimulation would be similar to that used in the fMRI experiments. The gratings were presented during the stimulus intervals, and a uniform gray field with the same mean luminance was presented during the blank intervals. The same gray field was presented between trials and during the initial $500 \mathrm{msec}$ fixation interval.

Moving gratings were presented on half of the trials, and counterphase gratings were presented on the other half of the trials. For moving grating trials, the direction of motion was reversed every second so that the first, third, and fifth seconds contained motion in one direction, and the second, fourth, and sixth seconds contained motion in the opposite direction. The direction of motion was chosen from eight possible directions $\left(0-315^{\circ}\right.$ at $45^{\circ}$ steps $)$. Counterphase gratings were chosen from four possible orientations $\left(0,45,90\right.$, or $\left.135^{\circ}\right)$. One of these 12 stimulus conditions was chosen pseudorandomly for each successive trial.

Visual stimuli were generated using a Cambridge Research graphics board (VSG 2/3) and presented on a Nanao 17 inch Flexscan monitor (model T2-17ts, $60 \mathrm{~Hz}$ screen refresh), placed $57 \mathrm{~cm}$ away from the monkey.

Electrophysiology. While the monkey performed the fixation task, neuronal activity was recorded from areas MT and MST using parylenecoated tungsten microelectrodes (Micro Probe Inc., impedance 1-2 M $\Omega$ at $1 \mathrm{kHz}$ ). Electrical signals were amplified and filtered, and action potentials from single and multiunits were detected with a time resolution of 1 msec using a dual time-amplitude window discriminator (Bak Electronics). When multiunit activity alone was recorded, an "event" was considered to be any excursion of the voltage trace above a set threshold (this might correspond to an action potential from a single neuron or a signal from several superimposed spikes). The threshold was set by hand so that baseline activity (in the absence of a stimulus) was 50-100 events per second.

When single and multiunit activity was recorded simultaneously, separate time-amplitude windows were employed for each signal. The two windows were nonoverlapping in the range of amplitudes accepted, so that the single unit action potentials were excluded from the multiunit activity. For each site, the multiunit receptive field location and the "preferred" direction were first mapped using a random dot stimulus that was controlled interactively by the experimenter.

Our multiunit measurement is likely to reflect the activity of a small population of neurons near the tip of the recording electrode. In previous studies from our laboratories, multiunit event counts obtained by this method correlated well with the root-mean-square power of the local field potential measured from the same electrode. For numerous recording sites in MT, disparity tuning curves were computed from both the multiunit and local field potential data. Preferred disparities obtained from the two data sets were nearly identical (regression slope near unity, $r=0.93 ; n=258 ; p<0.0001)$, and the disparity tuning indices were highly correlated as well (regression slope near unity, $r=0.78 ; n=396$; $p<0.0001$ ) (G. C. DeAngelis and W. T. Newsome, unpublished results). Thus, although we chose to use the multiunit measurement in the current study, the same results would have been obtained from local field potential measurements.

In monkey $\mathrm{S}$, microelectrodes were advanced from the occipital lobe and penetrated MT tangentially. In monkey M, microelectrodes were advanced from the frontal lobe and penetrated MT roughly normal to the surface of the cortex. MT was identified based on its high percentage of direction-selective units, its characteristic topography, and the stereotyped sequence of gray matter, white matter, and sulci along the electrode tracks. We also recorded four sites from MST in monkey M. Identifying MST in monkey M was relatively easy because electrode penetrations advancing from the frontal lobe necessarily pass through MST on the dorsal-anterior bank of the superior temporal sulcus before entering MT on the ventral-posterior bank. We have no histological confirmation of the recording sites because both monkeys are currently being used in related experiments. Additional details regarding our physiological methods can be found in Britten et al. (1992).

All procedures used in this study conformed to guidelines established by the National Institutes of Health for the care and use of laboratory animals.

Data analysis and statistics. The absolute magnitude of the multiunit response is somewhat arbitrary because it depends on the precise placement of the electrode tip and on the particular threshold level used for selecting multiunit events (see above). Hence, we computed a normalized response for each trial as:

$$
\mathrm{NR}=\frac{\text { response }- \text { baseline }}{\text { baseline }} .
$$

The response for each trial was quantified as the total number of events recorded during the $5.8 \mathrm{sec}$ interval of stimulus presentation. The baseline activity was estimated as the total number of events recorded during the $300 \mathrm{msec}$ interval before stimulus onset. These normalized responses were then averaged across trials and across recording sites. One-tailed, paired $t$ tests were used to test the null hypothesis that the resulting mean response to moving gratings was no greater than the mean response to counterphase gratings.

Data set. We recorded from a total of 61 multiunit sites in MT (25 in monkey M and 36 in monkey S) and four sites in MST (all from monkey M). In addition we recorded 12 single units from MT of monkey S.

\section{RESULTS}

\section{Moving versus counterphase gratings}

In a motion-sensitive brain area lacking interactions between opposite motion directions, one would predict that the total neuronal activity evoked by a counterphase grating would be greater than that evoked by a single moving grating component; the two moving grating components that make up the counterphase grating would evoke responses in two separate subpopulations of direction-selective neurons. With motion opponency, on the other hand, each neuronal subpopulation would be suppressed by the component grating moving in its nonpreferred direction. If the suppression were strong enough, then the total neuronal activity (summed across both subpopulations) evoked by the counterphase grating would actually be less than that evoked by either of the moving grating components presented alone.

In our first set of human fMRI experiments, counterphase gratings were presented in alternation with moving gratings. If V1 exhibits little motion opponency, then we would expect the fMRI responses to first increase during the counterphase grating presentations and then decrease during the moving grating presentations. If MT + exhibits strong motion opponency, then we would expect the fMRI responses to first decrease and then increase. In other words, the temporal phase of the fMRI responses in V1 should be near $0^{\circ}$, whereas the temporal phase in MT+ should be near $180^{\circ}$.

The results, plotted in Figure 2, demonstrate strong motion 

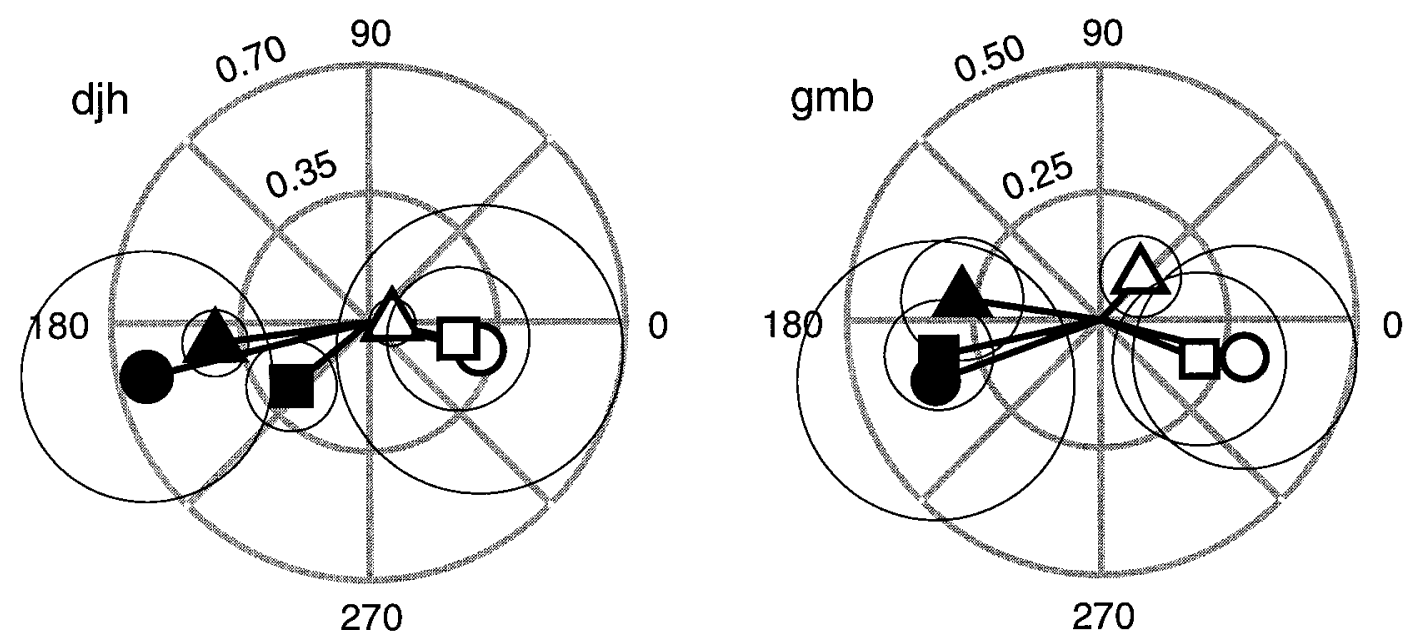

Figure 2. Motion opponency in human MT+. fMRI responses to stimuli that alternated between counterphase and moving gratings. Response amplitude (percent MR signal modulation) indicated by radial distance from the origin, and response temporal phase indicated by the angle from the horizontal axis. Responses from V1 (open symbols) are near $0^{\circ}$, in phase with the presentation of counterphase gratings. Responses from MT + ( filled symbols) are near $180^{\circ}$, in phase with the presentation of moving gratings. Two panels correspond to the two subjects. Plot symbols represent the vector average of between four and six measurements that were repeated in separate scans. Large circles represent $95 \%$ confidence intervals on the bivariate distributions of response amplitudes and phases. Circles: $\mathrm{sf}=0.8 \mathrm{cycle} /{ }^{\circ} ; \mathrm{tf}=4 \mathrm{~Hz}$; mean luminance $=36 \mathrm{~cd} / \mathrm{m}^{2}$; mean moving grating contrast $=$ $45.75 \%$; mean counterphase grating contrast $=91.5 \% ; n=5$ for both subjects. Squares: $\mathrm{sf}=0.4 \mathrm{cycle} /{ }^{\circ} ; \mathrm{tf}=8 \mathrm{~Hz} ;$ mean luminance $=3 \mathrm{~cd} / \mathrm{m}^{2} ;$ mean moving grating contrast $=44.25 \%$; mean counterphase grating contrast $=88.5 \% ; n=5$ for djh; $n=4$ for gmb. Triangles: sf $=0.8 \mathrm{cycle} /{ }^{\circ} ;$ tf $=4 \mathrm{~Hz}$; mean luminance $=36 \mathrm{~cd} / \mathrm{m}^{2}$; mean moving grating contrast $=6.25 \%$; mean counterphase grating contrast $=12.5 \% ; n=6$ for djh; $n=5$ for gmb.

opponency in $\mathrm{MT}+$. In the polar plots, fMRI response amplitudes are represented by the radial distances from the origin, and fMRI response phases are represented by the angles counterclockwise from the horizontal axis. For all three stimulus conditions, the fMRI responses in MT+ (filled symbols) were in phase with the presentation of moving gratings. In other words, MT+ brain activity was reduced by taking a single moving grating and superimposing a second grating moving in the opposite direction implying strong motion opponency. Superimposing the second grating increased the stimulus contrast, but nonetheless elicited a reduced response (circles, $p<0.001$ for djh and $p<0.05$ for gmb; squares, $p<0.001$ for djh and $p<0.01$ for gmb; triangles, $p<$ 0.001 for both subjects).

In V1 (open symbols), on the other hand, activity was slightly (although not always statistically significantly) increased by superimposing the second grating, implying little or no motion opponency on average. It is possible that a subset of direction-selective V1 neurons do exhibit motion opponency, but that our fMRI measurements reflect the lack of opponency in the majority of neurons.

To control for attentional state, subjects performed a contrast discrimination task throughout each fMRI scan (see Materials and Methods). Subjects reported that the task demanded more concentrated effort when it was made more difficult by reducing the contrast increments. For two of the conditions (circles and squares) the subjects' performance was somewhat better for counterphase gratings than for moving gratings (Table 1), suggesting that the subjects may have attended more to the moving gratings than to the counterphase gratings. In the third condition (triangles), we specifically adjusted the contrast increments to force subjects to attend more to the counterphase gratings than to the moving gratings (Table 1). The results (Fig. 2, triangles) again show greater MT+ activity to moving than to counterphase gratings, implying that any effect of attention is outweighed by motion opponency.
We ignored one repeat of the condition represented by the squares in Figure 2 for subject gmb. Although there was no obvious artifact in the functional MR images, this one repeat was clearly an outlier. The MT response (filled square) was 5.7 SDs (11.4 SEs) away from the mean of the other four repeats. The V1 response (open square) was 3.5 SDs (seven SEs) away from the mean of the other four repeats. With this scan included, the motion opponency effect in MT + falls below statistical significance threshold ( $p=0.06)$, but only for this one condition (Fig. 2 , filled square) in this one subject (gmb).

\section{Moving/counterphase gratings versus blank}

The data in Figure 2 indicate the differences in the fMRI signal amplitudes to the moving and counterphase gratings. These data cannot, however, be compared with electrophysiological measurements because a small amplitude could be caused by a small motion opponency effect, or it could be that the stimuli evoked only small responses to begin with.

We therefore conducted a separate experiment to quantify the magnitude of the effect by measuring responses to moving gratings and counterphase gratings separately. Figure 3 plots the results. MT+ responses were stronger for moving gratings (white bars) than for counterphase gratings (gray bars): $80 \%$ stronger for subject djh $(p<0.001)$ and $45 \%$ stronger for gmb $(p<0.01)$.

For neither subject was there a reliable difference in V1 activity, in spite of the contrast of the counterphase gratings being double that of the moving gratings. The lack of increase in activity with contrast in V1 might be caused by response saturation; the firing rates of individual V1 neurons typically saturate (level off) at high contrasts (see Discussion). However, it might also reflect a heterogeneity of responses with some individual neurons in V1 exhibiting decreased firing rates caused by motion opponency, whereas others were exhibiting increased firing rates with the increased contrast. 

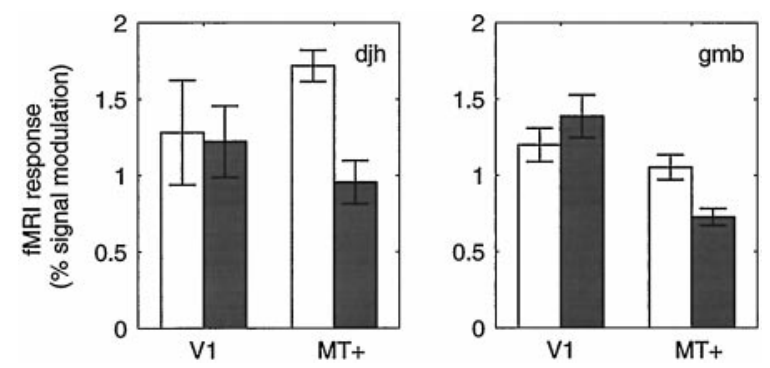

Figure 3. fMRI response amplitudes in visual areas V1 and MT+ to stimuli that alternated between test gratings and a uniform gray field. White bars, Moving gratings ( $\mathrm{sf}=0.4 \mathrm{cycle} /{ }^{\circ}$; $\mathrm{tf}=8 \mathrm{~Hz}$; mean luminance $=3 \mathrm{~cd} / \mathrm{m}^{2}$; mean contrast $=44.25 \% ; n=7$ for $\mathrm{djh} ; n=8$ for gmb). Gray bars, Counterphase gratings (same sf, tf, and mean luminance, mean contrast $=88.5 \% ; n=6$ for $\mathrm{djh} ; n=8$ for gmb). MT + responses are greater to moving than to counterphase gratings. Bar height, Mean component response amplitudes (see Materials and Methods). Error bars indicate SEM.

\section{Supersaturation control}

We performed a control experiment designed to rule out supersaturation of the responses as a possible alternative explanation of the results. fMRI measurements of brain activity in visual cortex typically increase monotonically with contrast and then saturate at high contrasts (Tootell et al., 1995a; Boynton et al., 1996, 1999; Demb et al., 1997, 1998). Using other techniques, however, researchers have sometimes observed supersaturation in which increasing the contrast of the stimulus beyond a certain level reduced response amplitudes (Li and Creutzfeldt, 1984; Tyler and Apkarian, 1985; Burr and Morrone, 1987; Bonds, 1991). Because the counterphase grating always had twice the contrast of the moving grating component, the possibility remains that increasing the contrast per se caused the decreased responses in Figure 3 and for the circles and squares in Figure 2 (supersaturation cannot explain the triangles in Fig. 2 because they correspond to low-contrast stimuli).

Figure 4 plots fMRI responses to moving gratings that alternated between high (mean, 95\%) contrast and medium (mean, $47.75 \%)$ contrast. There was no significant modulation of MT+ brain activity in either subject ( $p>0.8$ for djh; $p>0.12$ for gmb). For subject gmb the activity in V1 increased with contrast $(p<$ 0.01 ), but for subject djh the increase in V1 activity was not statistically significant. That is, the responses showed some evidence of response saturation in both brain areas, but no evidence of supersaturation at high contrasts.

Previously reported fMRI measurements as a function of contrast exhibit a clear dissociation between V1 and MT + . fMRI responses in MT + show extremely high gain at low contrasts and near complete saturation at high contrasts (Tootell et al., 1995a; Demb et al., 1998). Responses in V1 increase more gradually with contrast, roughly as a power law with an exponent of 0.3-0.4 (Tootell et al., 1995a; Boynton et al., 1996, 1999), a compressive nonlinearity that does not completely saturate at high contrasts. This is precisely the pattern of results evident in Figure 4 for subject gmb. When the contrast was increased from 47.75 to $95 \%$, V1 responses increased, but MT + responses were completely saturated. For subject djh, there was a tendency for the responses to increase with contrast both in V1 and in $\mathrm{MT}+$, although neither of these increases was statistically significant. It is entirely possible (given the size of the confidence intervals) that further repeated measurements in djh would reveal the usual dissociation between V1 and MT+ response saturation.
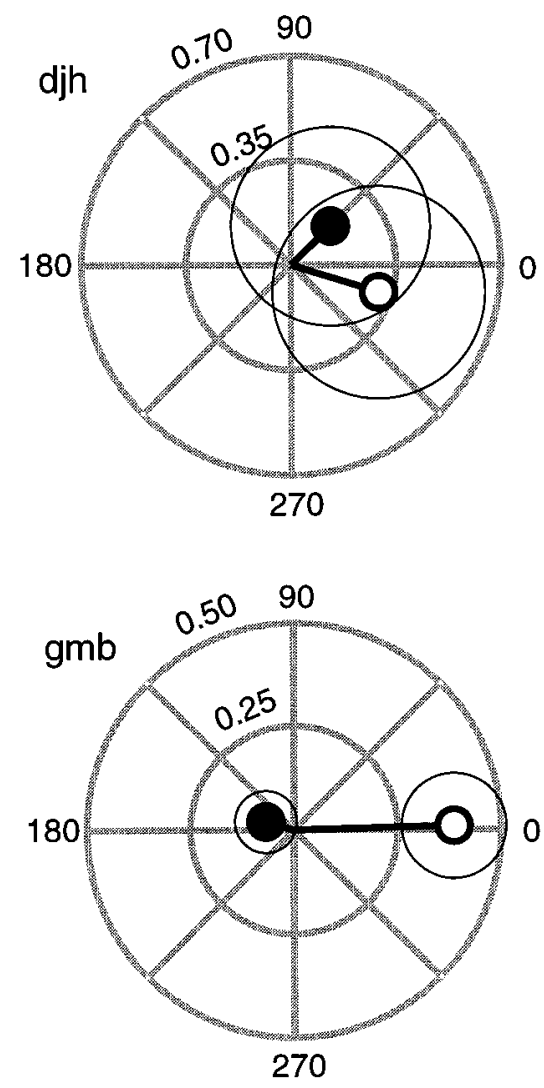

Figure 4. Supersaturation control. fMRI responses (same format as Fig. 2) to moving grating stimuli that alternated between high (mean, 95\%) and medium (mean, $47.75 \%$ ) contrasts. $\mathrm{sf}=0.8$ cycle $/{ }^{\circ}$; tf $=4 \mathrm{~Hz}$; mean luminance $=36 \mathrm{~cd} / \mathrm{m}^{2} ; n=6$ for djh; $n=5$ for $\mathrm{gmb}$.

\section{Paired versus unpaired dots}

Qian et al. (1994a) have demonstrated that humans perceive transparent motion in unpaired dot patterns, but that paired dots appear to flicker. This difference in the perception of motion to paired versus unpaired dot patterns was reflected in our experiments by the subjects' performance on the speed discrimination task. The speed increments for the paired dots were much larger than those for the unpaired dots, but the (percentage correct) performance of both subjects to both stimuli was about the same (Table 2).

The fMRI data, plotted in Figure 5, again show evidence for motion opponency in MT+. At both dot densities, and in both subjects, MT + activity (filled symbols) was in phase with the presentation of the unpaired dots. That is, MT + brain activity was reduced by pairing the dots ( $p<0.001$ for both subjects).

V1 responses, at the low dot density, were also reduced by pairing the dots ( $p<0.01$ for both subjects), but this was not the case at the high dot density. For subject gmb, V1 responses were increased by pairing the dots at the high dot density $(p<0.001)$. For subject djh, V1 responses were about equal for paired and unpaired dots at the high density. The results at the low dot density lead one to be concerned that the apparent motion opponency in MT + might simply be inherited from the V1 afferents. However, the dissociation between V1 and MT+ at the high dot density implies that this is not the case.

\section{Summary of fMRI results}

In the previous sections, we have seen that the fMRI signals in human V1 and MT + showed different levels of motion oppo- 


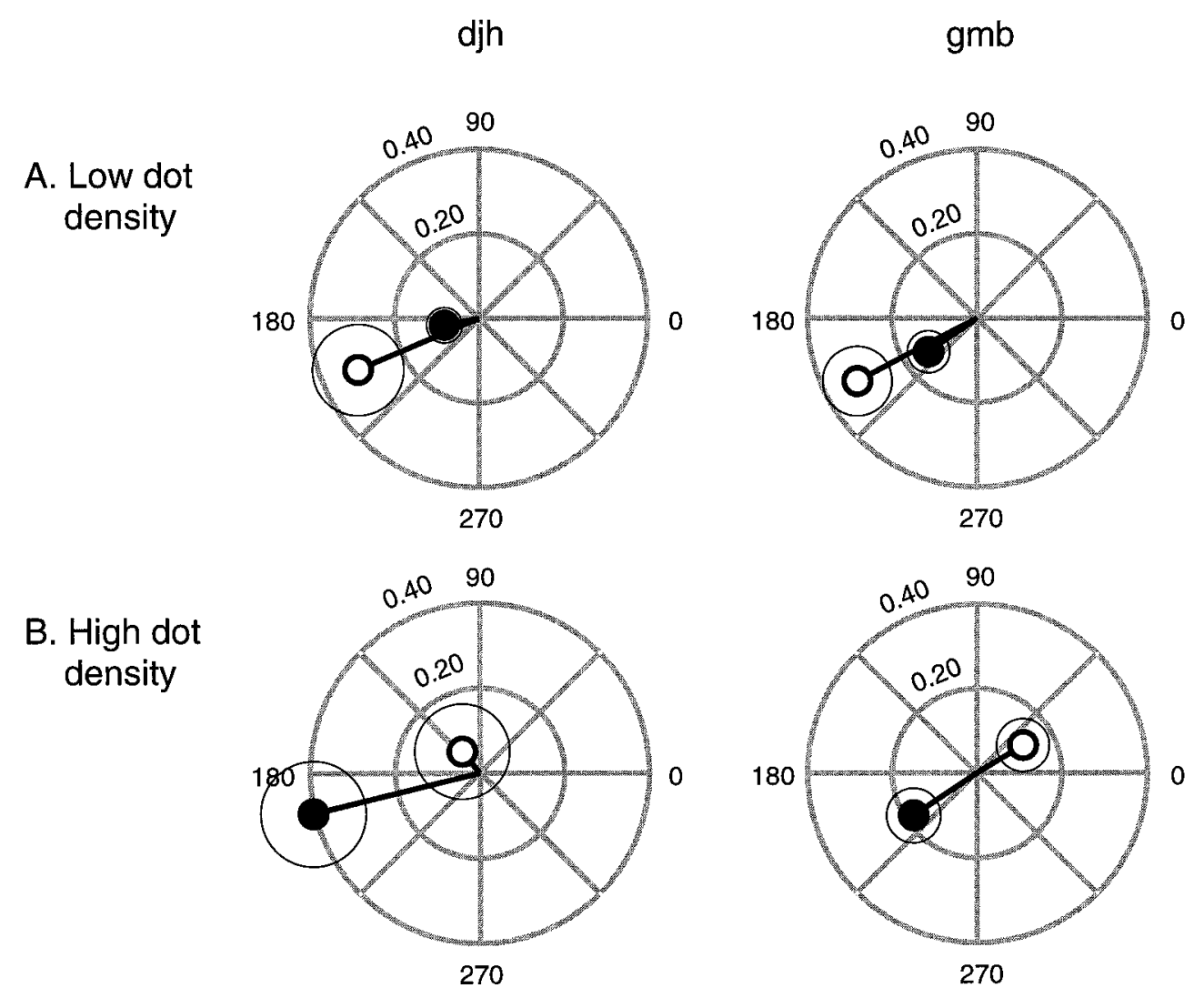

Figure 5. Motion opponency with random dot stimuli. fMRI responses (same format as Fig. 2 ) to paired versus unpaired dots. $A$, Low dot density $(n=$ 8 for both subjects). Both V1 (open symbols) and MT+ ( filled symbols) responses are near $180^{\circ}$, in phase with the presentation of unpaired dots. $B$, High dot density ( $n=8$ for both subjects). MT + responses ( filled symbols) are near $180^{\circ}$, in phase with the presentation of unpaired dots.

nency. Activity in human V1 was similar or slightly larger for moving gratings than for counterphase gratings. This agrees with previous electrophysiological measurements in cat and monkey primary visual cortex, and it agrees with computational models that have been used successfully to fit those electrophysiological measurements (see Discussion). Likewise, human V1 activity did not change consistently for paired versus unpaired dots. This agrees with previous electrophysiological experiments showing that the average response in macaque $\mathrm{V} 1$ to paired dot patterns is not significantly different from that to unpaired dot patterns (Qian and Andersen, 1994).

fMRI responses in human $\mathrm{MT}+$, on the other hand, consistently exhibited strong motion opponency. MT + responses were reduced for paired versus unpaired dots. This agrees with previous electrophysiological experiments showing that the average response in macaque MT is significantly reduced by pairing the dots (Qian and Andersen, 1994). Human MT+ responses were also reduced for counterphase versus moving gratings.

However, it is not clear from the published literature whether the average activity in monkey MT would be reduced by adding a pair of oppositely moving gratings. Consider, for example, presenting a rightward moving grating while recording from a rightward-selective MT column. Superimposing a leftward moving grating will typically reduce the net activity in this column, but it will simultaneously increase activity in an adjacent leftward-selective column. The key issue is the relative sizes of these two effects. For the macaque MT physiology to match the human MT+ fMRI, the reduction of activity in rightwardselective columns must exceed the increase in activity in leftward- selective columns, thus generating a net reduced response to the counterphase grating. We tested this prediction directly with MT multiunit recordings.

\section{Electrophysiology}

Figure 6 shows the response of a single MT multiunit site to moving and counterphase gratings. The top panel displays peristimulus time histograms (PSTHs) of responses to high contrast stimuli; the bottom panel shows responses to low-contrast stimuli. The six cycles of stimulus presentation can be seen clearly in the PSTHs. The dark solid curve depicts responses to a grating stimulus alternating between motion at $45^{\circ}$ (first) and motion at $225^{\circ}$ (second), whereas the light solid curve depicts responses to the reverse sequence $\left(225^{\circ}\right.$ first and $45^{\circ}$ second). The dashed curve shows the responses to a counterphase grating oriented at $135^{\circ}$ (the sum of the two moving gratings). For each of the six stimulus cycles, the $45^{\circ}$ moving grating (the "preferred" direction) elicited a stronger response than did the counterphase grating. In other words, the robust response to the preferred grating was reduced by superimposing a second grating moving in the opposite (or null) direction, as expected from motion opponency. On the other hand, the response to the counterphase grating was consistently stronger than the response to the null direction grating $\left(225^{\circ}\right)$. The same general trend is evident in response to the low-contrast stimuli (Fig. $6 B$ ).

Figure 7 summarizes the full data set obtained in the experiment of Figure 6 (see Materials and Methods). Figure $7 A$ plots the data obtained at high stimulus contrasts, whereas Figure $7 B$ plots the equivalent data set for low-contrast stimuli. The polar 
A

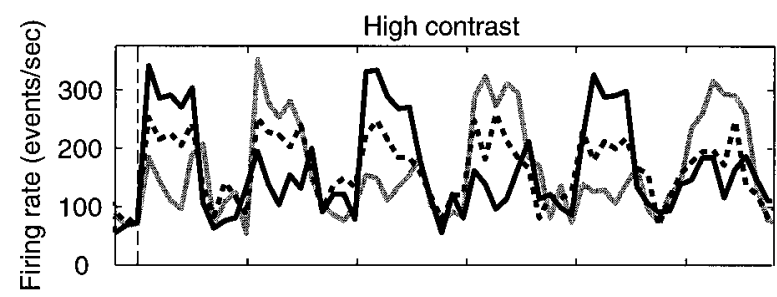

B

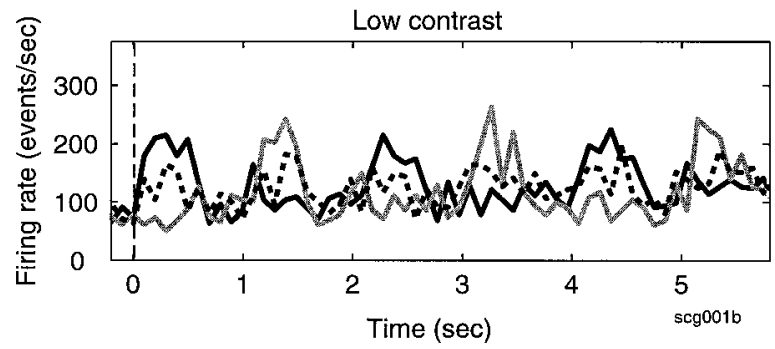

Figure 6. Multiunit responses in monkey MT to moving and counterphase grating stimuli shown as PSTH. $A$, Responses to high-contrast stimuli. $B$, Responses to low-contrast stimuli. Dark solid curves, Response to gratings moving first at $45^{\circ}$ and than at $225^{\circ}$. Light solid curves, Response to gratings moving first at $225^{\circ}$ and then at $45^{\circ}$. Dashed curves, Response to counterphase grating oriented at $135^{\circ}$. Responses are aligned to stimulus onset (time 0 ) and are binned in $100 \mathrm{msec}$ time bins.

plots depict average neuronal response as a function of direction of motion of single gratings (solid line) and response as a function of orientation of counterphase gratings (dashed line). The responses to the moving gratings in Figure 6 give rise to the data points at 45 and $225^{\circ}$ in Figure 7. The effects seen in the PSTHs of Figure 6 are apparent in their entirety in Figure 7. Adding the second grating to create a counterphase grating significantly reduced responses for three directions that responded well to the single grating stimulus and increased responses for one direction that responded poorly to the single grating. Responses at the two directions orthogonal to the preferred null axis (135 and $\left.315^{\circ}\right)$ were nearly unaffected.

The data in Figure 7 illustrate the responses of a single cluster of directional neurons to eight directions of motion and to four oriented counterphase gratings. If we assume that MT also contains matching clusters of neurons whose responses are similar to these, but which prefer other directions of motion, we can consider the recordings in Figure 7 to reflect the responses of eight different clusters of directional neurons to a single direction of motion and to a similarly oriented counterphase grating. We may infer from these data, therefore, that adding a second moving grating to produce a counterphase grating has two effects in MT: because of motion opponency, activity decreases in columns that respond well to the motion of the first grating, but activity increases in columns that respond poorly to the first grating. As indicated above, the key issue is the relative size of these two effects.

To assess this issue quantitatively, we compared the average response elicited at a given site by counterphase gratings to the average responses elicited by moving gratings. We computed this average across all four axes of motion employed in this experiment (e.g., Fig. 7). This procedure is necessary because the direction-tuning curves of MT neurons are broad, and any given stimulus elicits responses from neurons tuned to a variety of different directions. All of these neurons contribute, presumably,
A

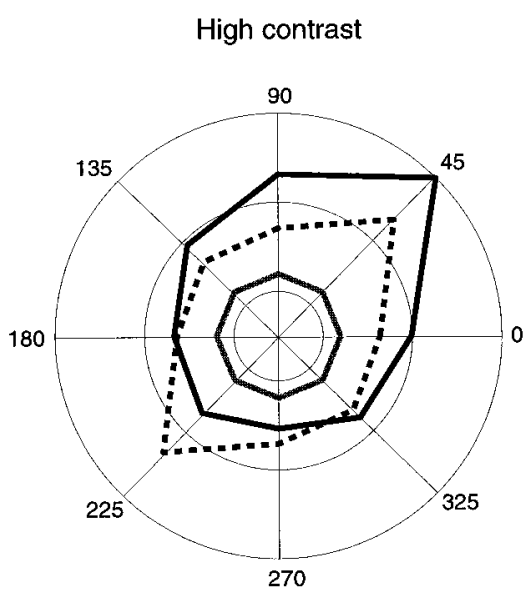

B

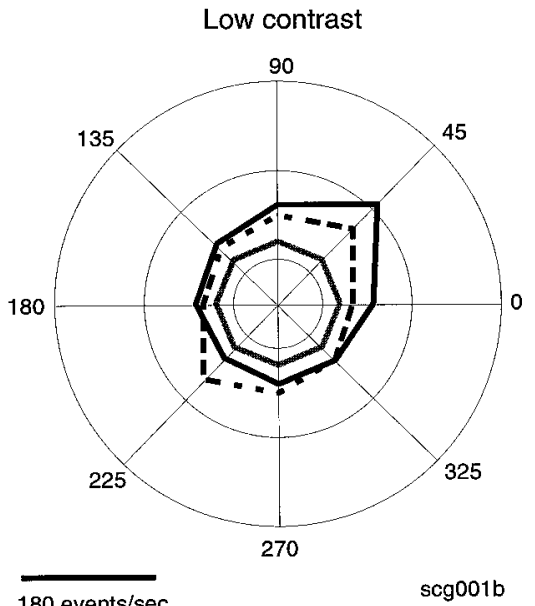

Figure 7. Polar plot depicting multiunit responses in monkey MT (same site as in Fig. 6) to moving and counterphase gratings. $A$, Responses to high-contrast stimuli. $B$, Responses to low-contrast stimuli. The angle of the polar plot indicates the direction of motion for the moving grating. For the counterphase gratings, each orientation is plotted twice at the two opposite directions from which the counterphase stimulus is composed (i.e., vertical counterphase stimulus generates two points at 0 and $180^{\circ}$ ). Radial distance from the origin indicates the magnitude of the response in events per second, scale given at the bottom left. The response to each stimulus condition was measured as the mean firing rate during the 500 msec starting $50 \mathrm{msec}$ after stimulus onset. Solid curves, Responses to moving stimuli. Dashed curves, Responses to counterphase stimuli. The gray polygon at the center indicates baseline activity estimated as the average firing rate during the $300 \mathrm{msec}$ preceding stimulus onset.

to the fMRI signal, which reflects changes in blood oxygenation over all of MT+. If macaque MT responds to the moving and counterphase gratings in a manner similar to human $\mathrm{MT}+$, the mean response to the counterphase gratings should be smaller, on average, than the mean response to the moving gratings, reflecting an overall reduction in neuronal activity.

In the experiment of Figure 7, the outcome of the analysis is consistent with the human fMRI data for both low- and highcontrast stimuli. For the high contrast (Fig. $7 A$ ), the mean response to single grating gratings was 95 events per second (after subtracting the baseline activity, see Materials and Methods), which is $24 \%$ greater than the mean response to counterphase gratings ( 76 events per second). For the low contrast (Fig. $7 B$ ), the mean response to moving gratings (41 events per second) was 


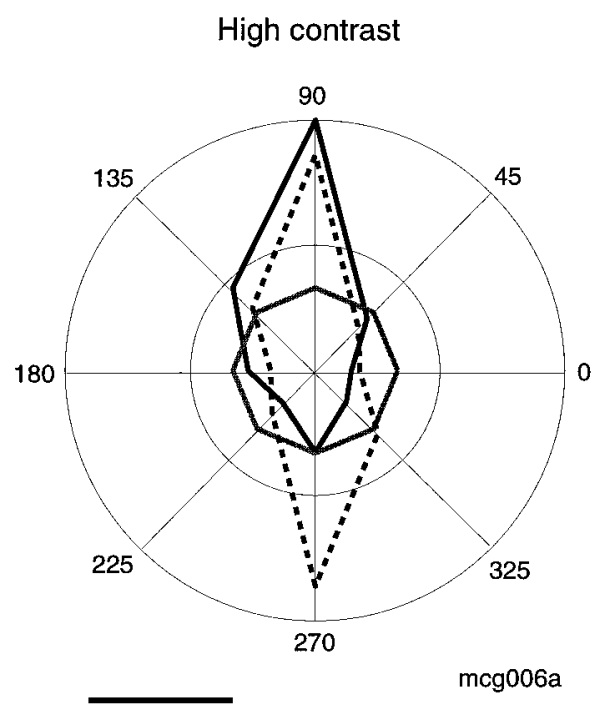

90 events/sec

Figure 8. Polar plot depicting multiunit responses (same format as Fig. 7) to high-contrast moving and counterphase gratings at a second site in monkey MT, which does not exhibit motion opponency.

$20 \%$ stronger than the mean response to counterphase gratings (34 events per second).

Although many MT sites yielded evidence of motion opponency, other sites showed little or no opponency, as illustrated in Figure 8 . Here, the response to a single grating moving in the preferred direction $\left(90^{\circ}\right)$ was reduced only slightly by the counterphase grating, whereas the response to a grating moving in the null direction $\left(270^{\circ}\right)$ was increased substantially. Averaged across the four axes of motion, the mean response to counterphase gratings at this site was actually stronger than the mean response to moving gratings.

The data in Figures 7 and 8 were computed from the stimulusevoked responses (i.e. only the $0.5 \mathrm{sec}$ intervals of stimulus presentations). In the fMRI study, however, the signal was averaged throughout periods that alternated between $0.5 \mathrm{sec}$ stimulus presentations and blank (uniform gray field) intervals (see Materials and Methods). Thus, a more direct comparison between the fMRI and electrophysiological data is to measure the overall activity in MT during the entire stimulus presentation, including the blank intervals. We therefore computed the mean neuronal activity over the entire trial duration $(5.8 \mathrm{sec})$, averaging as well over all stimulus directions (for moving gratings) or orientations (for counterphase gratings). The mean response was then normalized to the baseline activity (see Materials and Methods).

Figure 9 shows scatterplots of the mean normalized response to moving gratings ( $x$-axis) versus the mean normalized response to counterphase gratings ( $y$-axis) for the two monkeys. Figure $9 A$ depicts the normalized responses of all sites in monkey $\mathrm{S}$ for low contrast stimuli. Most sites lie below the diagonal line, indicating that the average response to moving gratings was stronger than the average response to counterphase gratings. The mean normalized response for moving gratings (0.32) was significantly stronger ( $p<0.001$; paired $t$ test) than the mean normalized response for counterphase gratings $(0.2)$. The result is similar at the high stimulus contrast (Fig. 9B), although the effect was somewhat weaker and more variable (means for moving and counterphase gratings were 0.6 and 0.53 , respectively; $p<0.005$,

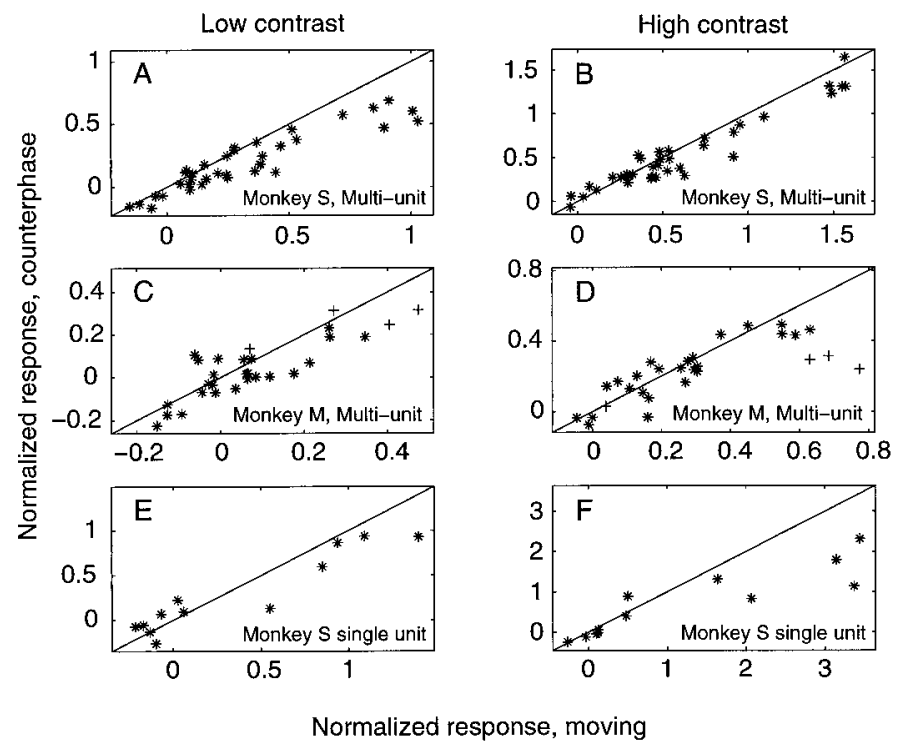

Figure 9. Scatter plot of the normalized responses to moving and counterphase stimuli in all sites recorded from the two monkeys. The response for each single or mulitunit site to moving stimuli was computed as the mean firing rate during the entire $5.8 \mathrm{sec}$ trial, averaged over all directions of motion. Similarly, the response to the counterphase stimuli was computed as the mean firing rate over all counterphase orientations. These responses were than normalized according to the baseline activity at that site (see Materials and Methods). $A$, Low-contrast stimuli in monkey S. $B$, High-contrast stimuli in monkey $\mathrm{S}$. $C$, Low-contrast stimuli in monkey $\mathrm{M}$. $D$, High-contrast in monkey $\mathrm{M}$. The four points marked by + correspond to the four MST sites. $E$, Normalized responses of 12 single units in MT of monkey $\mathrm{S}$ to low-contrast stimuli. $F$, Same for high contrast.

paired $t$ test). The results in monkey $\mathrm{M}$ were more noisy, but the overall pattern was the same (Fig. 9C,D). The mean responses to moving gratings, averaged across all recording sites, were significantly stronger than the mean responses to counterphase gratings $(p<0.05)$ at both high and low contrast (mean normalized responses for the high-contrast moving and counterphase gratings were 0.29 and 0.22 , respectively; means for the low-contrast moving and counterphase gratings were 0.078 and 0.042 , respectively). These results in monkey M, especially at the high contrast, were dominated by the three sites in MST (indicated by the plus signs) that showed pronounced motion opponency. More recordings would be necessary to determine whether the strong opponency interactions in these few sites reflect a genuine property of MST. It is important to note that both MT and MST are likely to contribute to the fMRI signal in human MT+.

Finally, Figure 9, E and F, shows the normalized responses of the 12 single units recorded from monkey S. Again, most points fall below the diagonal, even though there is great variability between units. The difference in the mean single-unit responses was statistically significant at the high stimulus contrast (mean normalized responses were 1.22 and 0.69 for moving and counterphase, respectively; $p<0.05$ ) but not at the low contrast (means were 0.35 and 0.28 for moving and counterphase, respectively; $p=0.25$ ).

Consistent with previous observations, our data suggest that motion opponency plays an important role in many, but not all sites in MT. More importantly for present purposes, the overall responses to counterphase gratings are smaller than those to single moving gratings, consistent with the outcome of the human fMRI experiments. 


\section{DISCUSSION}

\section{MT+, motion opponency, and direction selectivity}

The main result of this study is that we found evidence for motion opponency in human MT+. In MT + , brain activity elicited by a single moving grating was selectively reduced by superimposing a second grating moving in the opposite direction (Figs. 2, 3). Activity was not reduced when the two superimposed gratings moved in the same direction (Fig. 4). Such direction-specific interactions imply the presence of direction-selective signals. Specifically, the data are consistent with the notion that the two superimposed gratings evoke responses in two mutually inhibitory subpopulations of direction-selective neurons. Our data therefore support the notion that MT+, like monkey MT, processes directional signals, consistent with the conclusion reached by Tootell et al. (1995b) based on fMRI measurements of the motion aftereffect.

\section{MT+ and human motion perception}

The role of human MT+ in motion perception has been addressed previously using a variety of techniques. Patients with lesions that include this brain area show deficits in motion perception (Zihl and Cramon, 1983; Zihl et al., 1991; Vaina et al., 1994, 1998). Transcranial magnetic stimulation (TMS) near MT+ in healthy volunteers interferes with motion perception (Beckers and Hoemberg, 1992; Hotson et al., 1994; Beckers and Zeki, 1995). Functional neuroimaging studies have shown that MT+ is strongly activated when subjects view stimuli that appear to be moving (Zeki et al., 1991; Watson et al., 1993; McCarthy et al., 1995; Tootell et al., 1995a; Sereno et al., 1995; DeYoe et al., 1996; Smith et al., 1998), even for illusory motion in stationary displays (Zeki et al., 1993; Tootell et al., 1995b). Activity in MT+ can be modulated by instructing subjects to attend to moving stimuli (Corbetta et al., 1990; Beauchamp et al., 1997; O'Craven et al., 1997; Gandhi et al., 1999). MT+ responds selectively when subjects simply imagine visual motion stimuli (Goebel et al., 1998).

Our results provide additional evidence that activity in human $\mathrm{MT}+$ is correlated with the perception of motion. MT+ brain activity is reduced when a pair of superimposed gratings appears to flicker in place with no net motion (Figs. 2, 3). In addition, unpaired dot patterns that appear to move past one another like two semitransparent surfaces evoke greater activity in MT + than do paired dot patterns that appear to flicker (Fig. 5B).

\section{Modeling: divisive normalization and subtractive opponency}

Current computational theories provide a framework for interpreting our results. We focus here on a model recently proposed by Simoncelli and Heeger (1998), although other models share many of the same components.

The Simoncelli-Heeger model posits a particular kind of divisive suppression in V1 in which the response of each individual V1 neuron is divided by a quantity proportional to the summed activity of a large pool of neighboring neurons (Robson, 1988; Bonds, 1989; Albrecht and Geisler, 1991; DeAngelis et al., 1992; Heeger, 1992, 1993; Carandini and Heeger, 1994; Carandini et al., 1997; Nestares and Heeger, 1997; Tolhurst and Heeger, 1997a,b). The suppressive cortical neighborhood includes neurons with nearby receptive fields tuned for a range of orientations, directions, and spatiotemporal frequencies.

The Simoncelli-Heeger model also posits an additional subtractive inhibition in MT. The response of a velocity-selective MT neuron sums the responses of V1 afferents with compatible speed-direction preferences and subtracts the responses of V1 neurons with incompatible speed-direction preferences. For example, a model MT neuron tuned for up-rightward motion sums responses of V1 neurons tuned for up and right and subtracts responses of V1 neurons tuned for down and left.

Subtractive inhibition in MT predicts motion opponency and is, therefore, consistent with the key aspects of our results. According to the model, MT activity elicited by a moving grating should be reduced by superimposing a second grating moving in the opposite direction (Figs. 2, 3, 9). MT activity should also be reduced for paired versus unpaired dots (Fig. 5). According to some models, activity should be completely canceled by superimposing motions in opposite directions (Adelson and Bergen, 1985; van Santen and Sperling, 1985). Other models predict only partial opponency, i.e., that neuronal activity should be reduced but not abolished (Zemany et al., 1998). Our data, in both humans and monkeys, are more consistent with the latter.

Consistent with the model, V1 activity was similar or slightly larger for moving gratings than for counterphase gratings. The response of an individual V1 neuron to a grating moving in its preferred direction can be suppressed by superimposing a second grating moving in the opposite direction, as predicted by the divisive suppression in the model (Carandini et al., 1997; Tolhurst and Heeger, 1997a). However, superimposing the second grating also enhances the responses of other V1 neurons. According to the model, the sum total neuronal activity in V1 should depend only on the contrast energy of the stimulus, regardless of its motion. Increasing the contrast energy by superimposing a second grating should cause saturation of the sum total neuronal activity at high contrasts (consistent with the data in Fig. 4), but should never elicit a reduction in activity (consistent with the data in Figs. 2 and 3).

\section{Human fMRI versus macaque electrophysiology}

Previous studies have demonstrated the existence of motionopponent effects in monkey MT, but the consequences of opponency for the net activity of monkey MT (which presumably is the closest electrophysiological equivalent of the blood flow signal measured by fMRI) could not be inferred from previously published data. To determine whether superimposing a second moving grating would result in a net decrease in average brain activity in $\mathrm{MT}$, as we observed in human MT+ we recorded multiunit responses in areas MT and MST to moving and counterphase gratings similar to the ones employed in our fMRI study.

We were quite surprised to find that some MT recording sites (and single units) gave stronger average responses to counterphase than to moving gratings (Fig. 8). These sites (and single units) all showed motion opponency in the conventional sense that responses to a preferred direction were suppressed somewhat by superimposing a grating in the opposite direction. The suppression at these recording sites, however, was counterbalanced by the enhancement of the responses when superimposing the grating moving in the preferred direction on the nonpreferred stimuli, yielding stronger average responses to the counterphase than to the moving gratings. The variability in the degree of opponency between recording sites may reflect diversity between known subpopulations of MT neurons. The model proposed by Simoncelli and Heeger (1998), for example, predicts that motion opponency should be evident in the responses of so-called pattern motion-selective MT neurons but not in the responses of component motion-selective MT neurons (Movshon et al., 1986).

Despite the high variability in the electrophysiological mea- 
surements, when results from all sites and units were combined, we found that the average response was indeed suppressed in monkey MT as in human MT+. This general agreement between the monkey and human data provides further support for the homology between monkey MT and human MT+.

In addition, these results demonstrate that fMRI measurements are correlated with average spiking activity. The sequence of events from neuronal response to fMRI response is only partially understood (Malonek and Grinvald, 1996,1997; Buxton et al., 1998). Continuing to establish such links between fMRI signals in the human brain and the more familiar electrophysiological measurements in the monkey brain will be crucial in the further elaboration of human brain function.

\section{REFERENCES}

Adelson EH, Bergen JR (1985) Spatiotemporal energy models for the perception of motion. J Opt Soc Am 2:284-299.

Albrecht DG, Geisler WS (1991) Motion sensitivity and the contrastresponse function of simple cells in the visual cortex. Vis Neurosci 7:531-546.

Albright TD (1993) Cortical processing of visual motion. In: Visual motion and its role in the stabilization of gaze (Miles FA, Wallman J, eds), pp 177-201. Amsterdam: Elsevier.

Beauchamp MS, Cox RW, DeYoe EA (1997) Graded effects of spatial and featural attention on human area MT and associated motion processing areas. J Neurophysiol 78:516-520.

Beckers G, Homberg V (1992) Cerebral visual motion blindness: Transitory akinetopsia induced by transcranial magnetic stimulation of human area V5. Proc R Soc Lond B Biol Sci 249:173-178.

Beckers G, Zeki S (1995) The consequences of inactivating areas V1 and V5 on visual motion perception. Brain 118:49-60.

Belliveau JW, Kennedy Jr DN, McKinstry RC, Buchbinder BR, Weisskoff RM, Cohen MS, Vevea JM, Brady TJ, Rosen BR (1991) Functional mapping of the human visual cortex by magnetic resonance imaging. Science 254:716-719.

Bonds AB (1989) Role of inhibition in the specification of orientation selectivity of cells in the cat striate cortex. Vis Neurosci 2:41-55.

Bonds AB (1991) Temporal dynamics of contrast gain in single cells of the cat striate cortex. Vis Neurosci 6:239-255.

Boussaoud D, Ungerleider LG, Desimone R (1990) Pathways for motion analysis: cortical connections of the medial superior temporal and fundus of the superior temporal visual areas in the macaque. J Comp Neurol 296:462-495.

Boynton GM, Engel SA, Glover GH, Heeger DJ (1996) Linear systems analysis of fMRI in human V1. J Neurosci 16:4207-4221.

Boynton GM, Demb JB, Glover GH, Heeger DJ (1999) Neural basis of contrast discrimination. Vision Res 39:257-269.

Bradley DC, Qian N, Andersen RA (1995) Integration of motion and stereopsis in middle temporal cortical area of macaques. Nature 373:609-611.

Britten KH, Shadlen MN, Newsome WT, Movshon JA (1992) The analysis of visual motion: a comparison of neuronal and psychophysical performance. J Neurosci 12:4745-4765.

Burr DC, Morrone MC (1987) Inhibitory interactions in the human vision system revealed in pattern-evoked potentials. J Physiol (Lond) 389:1-21.

Buxton RB, Wong EC, Frank LR (1998) Dynamics of blood flow and oxygenation changes during brain activation: the balloon model. Magn Reson Med 39:855-864.

Carandini M, Heeger DJ (1994) Summation and division by neurons in primate visual cortex. Science 264:1333-1336.

Carandini M, Heeger DJ, Movshon JA (1997) Linearity and normalization of simple cells of the macaque primary visual cortex. J Neurosci 17:8621-8644.

Corbetta M, Miezin F, Dobmeyer S, Shulman G, Petersen S (1990) Attentional modulation of neural processing of shape, color, and velocity in humans. Science 248:1556-1559.

DeAngelis GC, Robson JG, Ohzawa I, Freeman RD (1992) The organization of suppression in receptive fields of neurons in the cat's visual cortex. J Neurophysiol 68:144-163.

DeYoe EA, Carman GJ, Bandettini P, Glickman S, Wieser J, Cox R
(1996) Mapping striate and extrastriate visual areas in human cerebral cortex. Proc Natl Acad Sci USA 93:2382-2386.

Demb JB, Boynton GM, Heeger DJ (1997) Brain activity in visual cortex predicts individual differences in reading performance. Proc Natl Acad Sci USA 94:13363-13366.

Demb JB, Boynton GM, Heeger DJ (1998) FMR imaging of early visual pathways in dyslexia. J Neurosci 18:6939-6951.

Dubner R, Zeki SM (1971) Response properties and receptive fields of cells in an anatomically defined region of the superior temporal sulcus of the monkey. Brain Res 35:528-532.

Engel SA, Rumelhart DE, Wandell BA, Lee AT, Glover GH, Chichilnisky E-J, Shadlen MN (1994) fMRI of human visual cortex. Nature 369:525.

Engel SA, Glover GH, Wandell BA (1997) Retinotopic organization in human visual cortex and the spatial precision of functional MRI. Cereb Cortex 7:181-192.

Gandhi SP, Heeger DJ, Boynton GM (1999) Spatial attention affects brain activity in human primary visual cortex. Proc Natl Acad Sci USA 96:3314-3319.

Glover GH, Lai S (1998) Self-navigating spiral fMRI: Interleaved versus single-shot. Magn Reson Med 39:361-368.

Goebel R, Khorram-Sefat D, Muckli L, Hacker H, Singer W (1998) The constructive natures of vision: direct evidence from functional magnetic resonance imaging studies of apparent motion and motion imagery. Eur J Neurosci 10:1563-1573.

Heeger DJ (1992) Normalization of cell responses in cat striate cortex. Vis Neurosci 9:181-198.

Heeger DJ (1993) Modeling simple cell direction selectivity with normalized, half-squared, linear operators. J Neurophysiol 70:1885-1898.

Hotson J, Braun D, Herzberg W, Boman D (1994) Transcranial magnetic stimulation of extrastriate cortex degrades human motion direction discrimination. Vision Res 34:2115-2123.

Krubitzer LA, Kaas JH (1990) Cortical connections of MT in four species of primates: areal, modular, and retinotopic patterns. Vis Neurosci 5:165-204.

Kwong KK, Belliveau JW, Chesler DA, Goldberg IE, Weiskoff RM, Poncelet BP, Kennedy DN, Hoppel BE, Cohen MS, Turner R, Cheng H-M, Brady TJ, Rosen BR (1992) Dynamic magnetic resonance imaging of human brain activity during primary sensory stimulation. Proc Natl Acad Sci USA 89:5675-5679.

Levinson E, Sekuler R (1975a) Inhibition and disinhibition of directionspecific mechanisms in human vision. Nature 254:692-694.

Levinson E, Sekuler R (1975b) The independence of channels in human vision selective for direction of movement. J Physiol (Lond) 250:347-366.

Li CY, Creutzfeldt O (1984) The representation of contrast and other stimulus parameters by single neurons in area 17 of the cat. Pflügers Arch 401:304-314.

Lubin J (1992) Interactions among motion-sensitive mechanisms in human vision. PhD thesis, University of Pennsylvania.

Malonek D, Grinvald A (1996) Interactions between electrical activity and cortical microcirculation revealed by imaging spectroscopy: implications for functional brain mapping. Nature 272:551-554.

Malonek D, Grinvald A (1997) Vascular imprints of neuronal activity: relationships between the dynamics of cortical blood flow, oxygenation, and volume changes following sensory stimulation. Proc Natl Acad Sci USA 94:14826-14831.

Mather G, Moulden B (1983) Thresholds for movement direction: two directions are less detectable than one. Q J Exp Psychol 35A:513-518.

Maunsell JHR, Van Essen DC (1983) The connections of the middle temporal visual area (MT) and their relationship to a cortical hierarchy in the macaque monkey. J Neurosci 3:2563-2586.

McCarthy G, Spicer M, Adrignolo A, Luby M, Core J, Allison T (1995) Brain activation associated with visual motion studied by functional magnetic resonance imaging in humans. Hum Brain Mapp 2:235-243.

Movshon JA, Newsome WT (1996) Visual response properties of striate cortical neurons projecting to area MT in macaque monkeys. J Neurosci 16:7733-7741.

Movshon JA, Adelson EH, Gizzi MS, Newsome WT (1986) The analysis of moving visual patterns. In: Experimental brain research supplementum II: pattern recognition mechanisms (Chagas C, Gattass R, Gross C, eds), pp 117-151. New York: Springer.

Nestares O, Heeger DJ (1997) Modelling the apparent frequencyspecific suppression in simple cells responses. Vision Res 37:1535-1543.

Newsome WT, Wurtz RH, Komatsu H (1988) Relation of cortical areas 
MT and MST to pursuit eye movements. II. Differentiation of retinal from extraretinal inputs. J Neurophysiol 60:604-620.

Noll D, Cohen J, Meyer C, Schneider W (1995) Spiral k-space MR imaging of cortical activation. J Magn Reson Imaging 5:49-57.

O’Craven KM, Rosen BR, Kwong KK, Treisman A, Savoy RL (1997) Voluntary attention modulates fMRI activity in human MT-MST. Neuron 18:591-598.

Ogawa S, Lee TM, Kay AR, Tank DW (1990) Brain magnetic resonance imaging with contrast dependent on blood oxygenation. Proc Natl Acad Sci USA 87:9868-9872.

Ogawa S, Tank DW, Menon R, Ellermannm JM, Kim S-G, Merkle H, Ugurbil K (1992) Intrinsic signal changes accompanying sensory stimulation: functional brain mapping with magnetic resonance imaging. Proc Natl Acad Sci USA 89:5951-5955.

Qian N, Andersen RA (1994) Transparent motion perception as detection of unbalanced motion signals: II. Physiology. J Neurosci 14:7367-7380.

Qian N, Andersen RA, Adelson EH (1994a) Transparent motion perception as detection of unbalanced motion signals: I. Psychophysics. J Neurosci 14:7357-7366.

Qian N, Andersen RA, Adelson EH (1994b) Transparent motion perception as detection of unbalanced motion signals: III. Modeling. J Neurosci 14:7381-7392.

Raymond J, Braddick O (1996) Responses to opposed directions of motion: continuum or independent mechanisms? Vision Res 36:1931-1937.

Robson JG (1988) Linear and nonlinear operations in the visual system. Invest Ophthalmol Vis Sci [Suppl] 29:117.

Sawyer-Glover AM, Glover GH (1998) fMRI of the motor cortex: comparison of EPI and spiral pulse sequences. Proceedings of SMRT Seventh Annual Meeting Sydney.

Schneider W, Noll DC, Cohen JD (1993) Functional topographic mapping of the cortical ribbon in human vision with conventional MRI scanners. Nature 365:150-153.

Sereno MI, Dale AM, Reppas JB, Kwong KK, Belliveau JW, Brady TJ, Rosen BR, Tootell RBH (1995) Borders of multiple visual areas in humans revealed by functional magnetic resonance imaging. Science 268:889-893.

Simoncelli EP, Heeger DJ (1998) A model of neural responses in visual area MT. Vision Res 38:743-761.

Smith AT, Greenlee MW, Singh KD, Kraemer FM, Hennig J (1998) The processing of first- and second-order motion in human visual cortex assessed by functional magnetic resonance imaging (fMRI). J Neurosci 18:3816-3830.

Snowden RJ, Treue S, Erikson RG, Andersen RA (1991) The response of area MT and V1 neurons to transparent motion. J Neurosci 11:2768-2785

Stromeyer CF, Kronauer RE, Madsen JC, Klein SA (1984) Opponentmovement mechanisms in human vision. J Opt Soc Am A 1:876-884.

Tolhurst DJ, Heeger DJ (1997a) Contrast normalization and a linear model for the directional selectivity of simple cells in cat striate cortex. Vis Neurosci 14:19-26.
Tolhurst DJ, Heeger DJ (1997b) Comparison of contrast normalization and threshold models of the responses of simple cells in cat striate cortex. Vis Neurosci 14:293-310.

Tootell RBH, Taylor JB (1995) Anatomical evidence for MT and additional cortical visual areas in humans. Cereb Cortex 5:39-55.

Tootell RBH, Reppas JB, Kwong KK, Malach R, Born RT, Brady TJ, Rosen BR, Belliveau JW (1995a) Functional analysis of human MT and related visual cortical areas using magnetic resonance imaging. J Neurosci 15:3215-3230.

Tootell RBH, Reppas JB, Dale AM, Look RB, Sereno MI, Malach R, Brady TJ, Rosen BR (1995b) Visual motion aftereffect in human cortical area MT revealed by functional magnetic resonance imaging. Nature 375:139-141.

Tyler CW, Apkarian PA (1985) Effects of contrast, orientation and binocularity in the pattern evoked potential. Vision Res 6:755-766.

Ungerleider LG, Desimone R (1986) Cortical connections of visual area MT in the macaque. J Comp Neurol 248:190-222.

Vaina LM, Grzywacz NM, Kikinis R (1994) Segregation of computations underlying perception of motion discontinuity and coherence. NeuroReport 5:2289-2294.

Vaina LM, Makris N, Kennedy D, Cowey A (1998) The selective impairment of the perception of first-order motion by unilateral cortical brain damage. Vis Neurosci 15:333-348.

van Santen JPH, Sperling G (1984) Temporal covariance model of human motion perception. J Opt Soc Am A 1:451-473.

van Santen JPH, Sperling G (1985) Elaborated Reichardt detectors. J Opt Soc Am A 2:300-321.

Watson AB, Thompson PG, Murphy BJ, Nachmias J (1980) Summation and discrimination of gratings moving in opposite directions. Vision Res 20:341-347.

Watson JDG, Myers R, Frackowiak RSJ, Hajnal JV, Woods RP, Mazziotta JC, Shipp S, Zeki S (1993) Area V5 of the human brain: evidence from a combined study using positron emission tomography and magnetic resonance imaging. Cereb Cortex 3:79-94.

Zeki S (1974) Functional organization of a visual area in the posterior bank of the superior temporal sulcus of the rhesus monkey. J Physiol (Lond), 236:549-573.

Zeki S, Watson JDG, Lueck CJ, Friston KJ, Kennard C, Frackowiak, RSJ (1991) A direct demonstration of functional specialization in human visual cortex. J Neurosci 11:641-649.

Zeki S, Watson JDG, Frackowiak RSJ (1993) Going beyond the information given: The relation of illusory motion to brain activity. Proc R Soc Lond B Biol Sci 252:215-222.

Zemany L, Stromeyer CF, Chaparro A, Kronauer RE (1998) Motion detection on flashed, stationary pedestal gratings: evidence for an opponent-motion mechanism. Vision Res 38:795-812.

Zihl J, Cramon DV (1983) Selective disturbance of movement vision after bilateral brain damage. Brain 106:313-340.

Zihl J, Cramon DV, Mai N, Schmid CH (1991) Disturbance of movement vision after bilateral posterior brain damage: further evidence and follow up observations. Brain 114:2235-2252. 